Network Working Group

Request for Comments: 2302

Category: Standards Track
G. Parsons

Northern Telecom

J. Rafferty

Human Communications

S. Zilles

Adobe Systems, Inc. March 1998

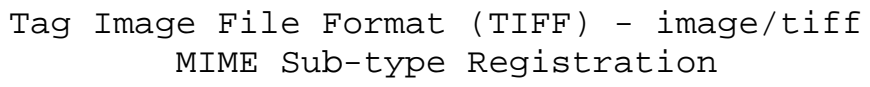

Status of this Memo

This document specifies an Internet standards track protocol for the Internet community, and requests discussion and suggestions for improvements. Please refer to the current edition of the "Internet Official Protocol Standards" (STD 1) for the standardization state and status of this protocol. Distribution of this memo is unlimited.

Copyright Notice

Copyright (C) The Internet Society (1998). All Rights Reserved.

Overview

This document describes the registration of the MIME sub-type

image/tiff. The baseline encoding is defined by [TIFF].

Internet Fax Working Group

This document is a product of the IETF Internet Fax Working Group.

All comments on this document should be forwarded to the email

distribution list at <ietf-faxeimc.org>.

1. Abstract

This document describes the registration of the MIME sub-type image/tiff. The baseline encoding is defined by [TIFF]. This document refines an earlier sub-type registration in RFC 1528 [TPC.INT] .

2. TIFF Definition

TIFF (Tag Image File Format) Revision 6.0 is defined in detail by Adobe in [TIFF]. The documentation can be obtained from Adobe at: 
Adobe Developers Association

Adobe Systems Incorporated

345 Park Avenue

San Jose, CA 95110-2704

Phone: $+1-408-536-6000$

Fax: $\quad+1-408-537-6000$

A copy of this specification can also be found in:

ftp://ftp.adobe.com/pub/adobe/devrelations/devtechnotes/pdffiles/ tiff6.pdf

While a brief scope and feature description is provided in this section as background information, the reader is directed to the original TIFF specification [TIFF] to obtain complete feature and technical details.

\subsection{TIFF Scope}

TIFF describes image data that typically comes from scanners, frame grabbers, and paint- and photo-retouching programs. TIFF is not a printer language or page description language. The purpose of TIFF is to describe and store raster image data. A primary goal of TIFF is to provide a rich environment within which applications can exchange image data. This richness is required to take advantage of the varying capabilities of scanners and other imaging devices. Though TIFF is a rich format, it can easily be used for simple scanners and applications as well because the number of required fields is small.

\subsection{TIFF Features}

Some of the features of TIFF (from [TIFF]) are:

- TIFF is capable of describing bilevel, grayscale, palette-color, and full-color image data in several color spaces.

- TIFF includes a number of compression schemes that allow developers to choose the best space or time tradeoff for their applications.

- TIFF is designed to be extensible and to evolve gracefully as new needs arise.

- TIFF allows the inclusion of an unlimited amount of private or special-purpose information. 


\section{MIME Definition}

\section{1 image/tiff}

The image/tiff content-type was previously defined in RFC 1528 as containing TIFF 6.0 encoded image data, with specific reference made to a subset known as TIFF Class F. This document re-defines the original image/tiff definition to refer to all of the profiles and extensions that build on TIFF 6.0 [TIFF] encoded image data, consistent with existing practice for TIFF aware Internet applications. This definition is further enhanced by introducing the new "application parameter" (section 3.2) to enable identification of a specific subset of TIFF and TIFF extensions for the encoded image data.

\subsection{Application parameter}

There are cases where it may be useful to identify the application applicable to the content of an image/tiff body. Typically, this would be used to assist the recipient in dispatching a suitable rendering package to handle the display or processing of the image file. As a result, an optional "application" parameter is defined for image/tiff to identify a particular application's subset of TIFF and TIFF extensions for the encoded image data, if it is known. No values are defined in this document.

Example using a fictional value 'foo':

$$
\text { Content-type: image/tiff; application=foo }
$$

There is no default value for application, as the absence of the application parameter indicates that the encoded TIFF image is Baseline TIFF or that it is not necessary to identify the application. It is up to the recipient's implementation to determine the application (if necessary) and render the image to the user.

4. IANA Registration

To: ietf-typeseiana.org

Subject: Registration of Standard MIME media type image/tiff

MIME media type name: image

MIME subtype name: tiff

Required parameters: none

Parsons, et. al. 
Optional parameters: application

There is no format specified for the value of this parameter in addition to that specified by [MIME1]. Various applications of TIFF may define values as required. New values should be defined in standards track RFCs and the values should be registered with IANA, using the registration form included in Appendix A. There is no default value for application, as the absence of the application parameter indicates that the encoded TIFF image is Baseline TIFF or that it is not necessary to identify the application. It is up to the implementation to determine the application (if necessary) and render the image to the user.

Encoding considerations: Binary or Base-64 generally preferred

Security considerations:

TIFF utilizes a structure which can store image data and attributes of this image data. The fields defined in the TIFF specification are of a descriptive nature and provide information that is useful to facilitate viewing and rendering of images by a recipient. As such, the fields currently defined in the TIFF specification do not in themselves create additional security risks, since the fields are not used to induce any particular behavior by the recipient application.

TIFF has an extensible structure, so that it is theoretically possible that fields could be defined in the future which could be used to induce particular actions on the part of the recipient, thus presenting additional security risks, but this type of capability is not supported in the referenced TIFF specification. Indeed, the definition of fields which would include such processing instructions is inconsistent with the goals and spirit of the TIFF specification.

Interoperability considerations:

The ability of implementations to handle all the defined applications (or profiles within applications) of TIFF may not be ubiquitous. As a result, implementations may decode and attempt to display the encoded TIFF image data only to determine that the image cannot be rendered. The presence of the application parameter may aid in allowing this determination before dispatching for rendering. However, it 
should be noted that the parameter value is not intended to convey levels of capabilities for a particular application.

Published specification:

TIFF (Tag Image File Format) is defined in:

TIFF (TM) Revision 6.0 - Final - June 3, 1992

Adobe Developers Association

Adobe Systems Incorporated

345 Park Avenue

San Jose, CA 95110-2704

Phone: $+1-408-536-6000$

Fax: $\quad+1-408-537-6000$

A copy of this specification can be found in:

ftp://ftp.adobe.com/pub/adobe/devrelations/devtechnotes/pdff iles/tiff6.pdf

Applications which use this media type:

Imaging, fax, messaging and multi-media

Additional information:

Magic number (s):

II (little-endian) : 49494200 hex

MM (big-endian): $\quad 4 D$ 4D 0042 hex

File extension(s): .TIF

Macintosh File Type Code(s): TIFF

Person \& email address to contact for further information:

Glenn W. Parsons

Glenn.Parsons@Nortel.ca

James Rafferty

Jrafferty@worldnet . att. net

Stephen Zilles

szilles@adobe.com

Intended usage: COMMON

Change controller: Stephen Zilles

Parsons, et. al.

Standards Track

[Page 5] 
5. Authors' Addresses

Glenn W. Parsons

Northern Telecom

P.O. Box 3511, Station C

Ottawa, ON K1Y $4 \mathrm{H} 7$

Canada

Phone: +1-613-763-7582

Fax: $\quad+1-613-763-2697$

Email: Glenn.Parsons@Nortel.ca

James Rafferty

Human Communications

12 Kevin Drive

Danbury, CT 06811-2901

USA

Phone: +1-203-746-4367

Fax: +1-203-746-4367

Email: Jrafferty@worldnet.att.net

Stephen Zilles

Adobe Systems Inc.

Mailstop W14

345 Park Avenue

San Jose, CA 95110-2704

USA

Voice: $\quad+1-408-536-4766$

Fax: $\quad+1-408-536-4042$

Email: szilles@adobe.com

6. References

[MIME1] Freed, N. and N. Borenstein, "Multipurpose Internet Mail Extensions (MIME) Part One: Format of Internet Message Bodies", RFC 2045, November 1996.

[MIME4] Freed, N. and N. Borenstein, "Multipurpose Internet Mail Extensions (MIME) Part Four: Registration Procedures", RFC 2048, November 1996.

[TIFF] Adobe Developers Association, TIFF (TM) Revision 6.0 - Final, June 3, 1992 .

[TPC.INT] Malamud, C. and M. Rose, "Principles of Operation for the TPC.INT Subdomain: Remote Printing -- Technical Procedures", RFC 1528, October 1993.

[TIFFPLUS] McIntyre, L., Zilles, S., Buckley, R., Venable, D., Parsons, G., and J. Rafferty, "File Format for Internet Fax", RFC 2301, March 1998.

[TIFF] Parsons, G., and J. Rafferty, "Tag Image File Format TIFF) -- R Profile for Facsimile, RFC 2306, March 1998.

Parsons, et. al.

Standards Track

[Page 6] 
Appendix A: IANA Registration form for new values of Application Parameter

To: IANA@isi.edu Subject: Registration of new values for the Application parameter

$$
\text { of image/tiff }
$$

MIME type name:

image/tiff

Optional Parameter:

Application

New Value (s):

Application=foo

Description of Use:

foo - ("foo" is a fictional new value used in this message as an example, it is to be replaced with the new value being registered. Include a short description of the use of the new value here. This must include reference to a standards track RFC for the complete description; the use of the value must be defined completely enough for independent implementation. )

Security Considerations:

(Any additional security considerations that may be introduced by use of the new parameter should be defined here or in the referenced standards track RFC.)

Person \& email address to contact for further information:

(fill in contact information)

INFORMATION TO THE SUBMITTER:

The accepted registrations will be listed in the "Assigned Numbers" series of RFCs. The information in the registration form is freely distributable.

Parsons, et. al.

Standards Track 
Full Copyright statement

Copyright (C) The Internet Society (1998). All Rights Reserved.

This document and translations of it may be copied and furnished to others, and derivative works that comment on or otherwise explain it or assist in its implementation may be prepared, copied, published and distributed, in whole or in part, without restriction of any kind, provided that the above copyright notice and this paragraph are included on all such copies and derivative works. However, this document itself may not be modified in any way, such as by removing the copyright notice or references to the Internet society or other Internet organizations, except as needed for the purpose of developing Internet standards in which case the procedures for copyrights defined in the Internet Standards process must be followed, or as required to translate it into languages other than English.

The limited permissions granted above are perpetual and will not be revoked by the Internet society or its successors or assigns.

This document and the information contained herein is provided on an "AS IS" basis and THE INTERNET SOCIETY AND THE INTERNET ENGINEERING TASK FORCE DISCLAIMS ALL WARRANTIES, EXPRESS OR IMPLIED, INCLUDING BUT NOT LIMITED TO ANY WARRANTY THAT THE USE OF THE INFORMATION HEREIN WILL NOT INFRINGE ANY RIGHTS OR ANY IMPLIED WARRANTIES OF MERCHANTABILITY OR FITNESS FOR A PARTICULAR PURPOSE. 